\title{
Optimisation of red light-emitting diodes irradiance for illuminating mixed microalgal culture to treat municipal wastewater
}

\author{
K. Mohammed, Z. S. Ahammad, P. J. Sallis \& C. R. Mota \\ School of Civil Engineering and Geosciences, Newcastle University, UK
}

\begin{abstract}
This paper evaluates the effect of variation in red light-emitting diodes (LEDs) irradiance on the growth rate and biomass productivity of a mixed culture of microalgae grown on synthetic municipal wastewater, with and without $\mathrm{CO}_{2}$ addition. Red LEDs were used to illuminate microalgal culture from the centre of 21-L stirred-tank photobioreactors made of transparent Plexiglas, each reactor having a working volume of $16 \mathrm{~L}$. The reactors were operated in batch mode with $\mathrm{pH}$ control, and under continuous illumination for 30 days at ambient temperature. Mixing was achieved through the use of overhead mechanical stirrers operated at $100 \pm 1$ and $60 \pm 1$ revolution per minute, before and after the addition of $\mathrm{CO}_{2}$, respectively. Three average values of irradiance of 429.9, 582.7 and $730.8 \mu \mathrm{mol} . \mathrm{s}^{-1} . \mathrm{m}^{-2}$ were used to illuminate the reactors, with a control reactor operated in the dark. $\mathrm{CO}_{2}$ addition resulted in about two-fold increase in biomass productivity in all the experimental reactors. The bioreactor with medium irradiance yielded the highest biomass productivity and maximum specific growth rate of $0.034 \mathrm{~g} . \mathrm{L}^{-1} \cdot \mathrm{d}^{-1}$ and $0.109 \mathrm{~d}^{-1}$, respectively. The findings in this study show that both microalgal growth rate and biomass productivity are not always directly proportional to irradiance, despite the influence of process and operational parameters. Furthermore, a medium amount of irradiance resulted in optimum growth and productivity of the mixed microalgal culture. Keywords: microalgae, biomass productivity, growth rate, optimum irradiance, red light emitting diode, synthetic municipal wastewater.
\end{abstract}




\section{Introduction}

Amount and quality of illumination play a key role in the growth of aquatic photosynthetic organisms such as microalgae. These illumination requirements can influence the ability of microalgae to remove nutrients from wastewater. Conventional light sources such as fluorescent and incandescent lamps are widely used to externally illuminate microalgal photobioreactors (PBRs). However, such light sources have relatively high carbon footprint.

In addition, light attenuation resulting from long path length in PBRs, coupled with high algal concentration, can be one of the shortcomings of illuminating PBRs externally. Furthermore, visible light absorption and scattering by materials suspended in water column result in loss of light photons and consequently reduce the amount of photosynthetic active radiation reaching microalgal cells, leading to light limitation [1].

Internally illuminating microalgal PBRs with red light-emitting diodes can overcome the above shortcomings. LEDs emitting monochromatic light are recently gaining popularity in microalgal cultivation $[2,3]$. However, there is a need to optimise the quantity and quality of LED irradiance for illuminating microalgal systems. Nevertheless, optimum quantity of irradiance may differ from one system to another.

Therefore, such optimisation studies are prerequisite to the development of energy-efficient, carbon-neutral microalgal wastewater treatment technologies. LEDs can potentially replace conventional light sources due to the advantages of the former over the latter [4]. Such advantages include lower power consumption; lower input voltage; luminous efficacy [5]; lower start-up time; easy control; monochromatic property; and longer life span [4].

More importantly, LEDs have very low carbon footprint with associated opportunity for carbon credits as well as high potential towards enhancing environmental sustainability; they have lower carbon footprint than fluorescent and incandescent lamps [4]. Interestingly, used LEDs can be recycled easily as they are composed of fairly benign substances compared to incandescent lamps that contain mercury which poses higher pollution risk to the environment [4].

Furthermore, the problems of light limitation associated with PBR scale-up can be overcome through focussed research on the use of LEDs to replace conventional light sources. Due to their small size, many LEDs can be mounted on a narrow strip of matrix board and inserted into PBRs in a water-tight chamber (to prevent short-circuiting when in contact with water) in order to fully utilise their light output and consequently eliminate light limitation.

In this study, red LEDs emitting light at $660 \mathrm{~nm}$ were chosen as light source based on the premise that red photons are most efficient in deriving photosynthesis and that they are weakly absorbed by water molecules, leading to minimal loss of light due to absorption [1].

In addition, this wavelength was chosen based on the fact that it falls within the range of maximum photon absorption peaks in chlorophyll $a$ and $b$ molecules, the main photosynthetic pigments in green algae, i.e. 663 and $645 \mathrm{~nm}$; [1]. This would also facilitate optimal photon energy utilisation. 
Therefore, the aim of this study was to determine the optimum irradiance required to illuminate microalgal culture to treat municipal wastewater.

\section{Materials and methods}

\subsection{Bioreactor design}

Internally-illuminated stirred-tank type photobioreactor (STPBR) made of transparent Plexiglas was used in this study. Illumination was provided radially from the centre of the STPBRs by LEDs (Kingbright, Taiwan) emitting red light at $660 \mathrm{~nm}$ characteristic wavelength. The STPBR was $30 \mathrm{~cm}$ deep and $30 \mathrm{~cm}$ in diameter, with a total volume of about $21 \mathrm{~L}$. It has a central chamber for housing LEDs soldered onto matrix boards, here referred to as 'LED core'.

The LED core was designed and constructed separate from the central chamber for easy maintenance. The PBR has a light path of $11 \mathrm{~cm}$ and a surfaceto-volume ratio of about $0.14 \mathrm{~cm}^{-1}$. It was also designed to illuminate the PBR up to a maximum water depth of $28 \mathrm{~cm}$ and allow degassing head space of $2 \mathrm{~cm}$. Three STPBRs (R1, R2 and R3) with different irradiance values were used in the study. A similar reactor operated in the dark (R0), served as a control. Power was supplied to the LEDs using AC-DC adaptors (RS Components, UK) while mixing was provided using an overhead mechanical stirrer (IKA, UK).

\subsection{Experimental set-up}

\subsubsection{Irradiance}

All the reactors were covered with aluminium foil to concentrate light in the PBRs and to isolate the control reactor maintain it in the dark. Irradiance was measured with LI-192 Underwater Quantum Sensor connected to LI-250 light meter (LI-COR Biosciences, USA). Light measurements were performed in air, distilled water, and microalgal culture, at varying distance beginning from the wall of the LED core to at least the maximum PBR light path length. The average irradiance values used to illuminate the PBRs are presented in Table 1.

Table 1: $\quad$ Average irradiance supplied to the reactors.

\begin{tabular}{ccc}
\hline Reactor & Irradiance $\left(\mu\right.$ mol. $\left.\mathrm{s}^{-1} \cdot \mathrm{m}^{-2}\right)$ & Relative category \\
\hline R0 & 0 & - \\
R1 & $429.9 \pm 0.81$ & Low \\
R2 & $582.7 \pm 3.16$ & Medium \\
R3 & $730.8 \pm 1.52$ & High \\
\hline
\end{tabular}

\subsubsection{Synthetic municipal wastewater and microalgal inoculum}

Synthetic municipal wastewater [6], modified to mimic an anaerobically digested wastewater, was used in the study. It was autoclaved at $120^{\circ} \mathrm{C}$ for 15 minutes (Rodwell Scientific Instruments, UK) and allowed to cool at room temperature prior to its use in the experiment. The wastewater was inoculated with mixed 
microalgal culture. The algal culture was obtained from a previous bench-scale laboratory experiment. It was centrifuged at $1000 \mathrm{~g}$ for 20 minutes at $22^{\circ} \mathrm{C}$ and maintained in $1 \mathrm{~L}$ Pyrex beaker under red LED illumination and agitated with a magnetic stirrer (Hanna Instruments, UK) prior to the experiments. The wastewater was prepared and inoculated in a big container from which $16 \mathrm{~L}$ was supplied to each reactor.

\subsubsection{Reactor operation and monitoring}

All the reactors were operated in batch mode with $\mathrm{pH}$ control at ambient condition and under continuous illumination [7, 8], for 30 days. Hydrochloric acid $(\mathrm{HCl})$ and sodium hydroxide $(\mathrm{NaOH})$ were appropriately used to control $\mathrm{pH}$ within the range of 7.0 to 8.5 in all the reactors and overhead mechanical stirrers operated at $60 \pm 1$ and $100 \pm 1$ revolution per minute, corresponding to presence and absence of $\mathrm{CO}_{2}$ addition, respectively, were used to mix the content of the reactors.

Premixed industrial-grade gas composing of $10 \% \mathrm{CO}_{2}, 6 \% \mathrm{O}_{2}$ and $84 \% \mathrm{~N}_{2}$ (BOC Gas, UK) was used in the study. $\mathrm{CO}_{2}$ addition into the reactors began on the 24th day of the experiment, corresponding to the lowest concentration of inorganic carbon in the mixed liquor. The gas was supplied continuously into each reactor through silicon tubing connected to rotameters (RS Components, UK) which were thence terminally connected to $0.5 \mathrm{~mm}$ pore gas spargers (Science Laboratory Supplies, UK). The $\mathrm{CO}_{2}$ was supplied into each reactor at a flow rate of $100 \mathrm{~mL}$ per minute and lasted for $7 \mathrm{~d}$. Samples were collected every $24 \mathrm{~h}$ for the first week and subsequently every $48 \mathrm{~h}$ for physico-chemical analyses.

\subsection{Analytical tests}

Samples collected from the reactors were chemically analysed for ammonium $\left(\mathrm{NH}_{4}-\mathrm{N}\right)$; chemical oxygen demand (COD); total Kjeldahl nitrogen (TKN); anions: nitrite $\left(\mathrm{NO}_{2}-\mathrm{N}\right)$, nitrate $\left(\mathrm{NO}_{3}-\mathrm{N}\right)$ and phosphate $\left(\mathrm{PO}_{4}{ }^{3-}\right)$; and total carbon (TC). In addition, optical density (OD) and cell dry weight (CDW) of the mixed microalgal culture, $\mathrm{pH}$, dissolved oxygen (DO) and temperature were also measured. $\mathrm{COD}$ and $\mathrm{NH}_{4}-\mathrm{N}$ were, respectively, measured using commercial test kits (Merck, Germany) and a spectrophotometer (spectroquant, Merck, Germany) based on manufacturer's instructions and according to Standard Methods [9].

In addition, anions were determined using ion chromatography with ICS 1000 connected to conductivity detector and anion column (with eluent composing of $1 \mathrm{mM} \mathrm{NaHCO}_{3}$ and $8 \mathrm{mM} \mathrm{Na}_{2} \mathrm{CO}_{3}$ at a flow rate of $1 \mathrm{~mL} \mathrm{~min}^{-1}$ ) whereas TC was determined using automated total organic carbon analyser (Shimadzu, Japan). Dissolved oxygen (DO) and temperature were measured using a potable DO meter (VWR, UK) and $\mathrm{pH}$ was monitored using $\mathrm{pH}$ meter (Jenway, UK). Furthermore, microalgal $\mathrm{CDW}$ and $\mathrm{OD}$ were measured using gravimetry and UV-1700 spectrophotometer (Shimadzu, Japan), respectively. All samples for chemical analyses were filtered through $0.2 \mu \mathrm{m}$ filters (Sartorius, UK) prior to measurements and analyses were performed in replicate except for $\mathrm{pH}$. 


\section{Results and discussion}

\subsection{Light measurement}

The study began with evaluation of light attenuation which might affect performance of the STPBRs (Fig. 1) since exposure of microalgal cells to sufficient light photons is essential for growth.

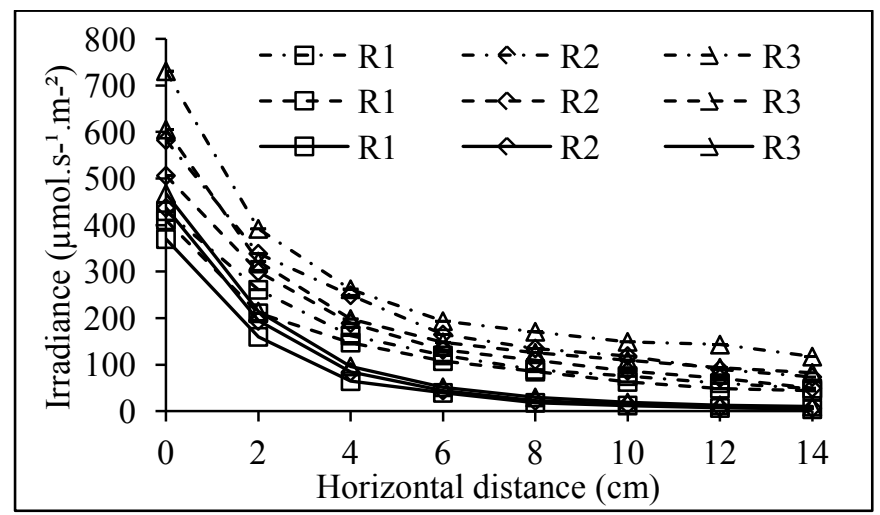

Figure 1: Irradiance versus horizontal distance in the STPBRs (dash dot, in air; dash, distilled water; continuous, $82 \mathrm{mg}$ microalgal CDW L-1).

For all the media used in the light measurement, irradiance exponentially decreased with increasing horizontal distance in the STPBRs (Fig. 1). Interestingly, the irradiance on the wall of the LED core decreased with increasing media densities; having a highest value in air and the lowest in the algal culture. This suggests that the density of a medium primarily promotes the absorption of light photons independent of the distance from the source. The choice of the initial microalgal concentration of the experimental culture was based on the evaluation of the light attenuation with respect to the microalgal CDW shown in Fig. 1 and other CDW values (data not shown).

\subsection{Specific growth rate, biomass productivity and optimum irradiance}

Microalgal specific growth rates were calculated based on the variation of CDW with time, in natural logarithm, while biomass productivity was computed from CDWs as the amount of microalgal biomass produced daily. Both the growth rate and productivity increased with increasing irradiance up to $582.7 \mu \mathrm{mol} \mathrm{s}^{-1} \mathrm{~m}^{-2}$, with subsequent decline up to the maximum irradiance.

Maximum specific growth rate and biomass productivity of $0.109 \mathrm{~d}^{-1}$ and $0.034 \mathrm{~g} \mathrm{~L}^{-1} \mathrm{~d}^{-1}$, respectively, were achieved at medium irradiance. This suggests that the medium irradiance was optimum for microalgal growth under the experimental conditions. However, the relatively low values in both growth rate and biomass productivity might be due to inorganic carbon limitation observed 
in the experimental reactors prior to $\mathrm{CO}_{2}$ addition. This might have masked the effect of inorganic carbon supplementation on the microalgal culture.

Interestingly, lack of illumination has limited microalgal growth in the control reactor irrespective of the available organic carbon which may favour algal heterotrophic metabolism. This suggests the dominance of obligate photoautotrophs in the microalgal culture [10].

\subsection{Wastewater treatment efficiency}

Reactor performance with respect to wastewater treatment was evaluated in terms of COD and nutrient removal efficiencies. COD removal efficiency varied with time in all the reactors with maximum of about $82 \%$ recorded at high irradiance. This suggests that COD removal does not directly depend on irradiance. Interestingly, removal efficiency of up to $79 \%$ was also achieved in the control reactor, suggesting the presence of bacteria in the mixed microalgal culture as algae are not directly responsible for COD removal $[11,12]$.

Noteworthy, greater COD removal was achieved with $\mathrm{CO}_{2}$ addition compared to operating the reactors without $\mathrm{CO}_{2}$ addition. This apparently shows the direct effect of inorganic carbon supplementation on microalgal growth with consequent favourable condition for bacterial degradation of organic matter with the aid of photosynthetic oxygenation.

\subsection{Nitrite accumulation}

Nitrite concentrations were recorded in the illuminated reactors with maximum of up about $50 \mathrm{mg} \mathrm{NO}-\mathrm{N} \mathrm{L}^{-1}$ in the PBR operated at high irradiance. Interestingly, higher concentration of about $88 \mathrm{mg} \mathrm{NO}_{2}-\mathrm{N} \mathrm{L}^{-1}$ was recorded in the control reactor. The $\mathrm{NO}_{2}-\mathrm{N}$ concentration consistently accumulated with time and appeared to be light-dependent as it decreased with increasing irradiance.

This suggests partial nitrification of $\mathrm{NH}_{4}-\mathrm{N}$ in the mixed liquor. However, high concentration of nitrite have been reported of possibly posing toxic effects on aquatic organisms, especially under non-steady-state condition [13], which may usually be the case with systems operated in batch mode.

\section{Conclusion}

$\mathrm{CO}_{2}$ addition to the mixed microalgal culture treating synthetic municipal wastewater resulted in about two-fold increase in biomass productivity in all the STPBRs with the reactor operated at medium irradiance exhibiting the highest specific growth rate and biomass productivity of $0.109 \mathrm{~d}^{-1}$ and $0.034 \mathrm{~g} \mathrm{~L}^{-1} \mathrm{~d}^{-1}$, respectively.

However, inorganic carbon limitation observed in the experimental reactors prior to $\mathrm{CO}_{2}$ addition might have limited the growth and productivity of the mixed microalgal culture which might have consequently affected wastewater treatment efficiency.

In addition, nitrite accumulation might have posed toxic effects on the microbial population and possibly masked the effect of inorganic carbon 
supplementation on the microalgal growth. The findings in this study suggest that both microalgal growth rate and biomass productivity are not always directly proportional to irradiance. Furthermore, medium amount of irradiance resulted in optimum growth and productivity of the mixed microalgal culture.

\section{Acknowledgements}

The authors are grateful to Petroleum Technology Development Fund, Abuja, Nigeria, for awarding a PhD Scholarship to Mr K Mohammed. Mr Rob Hunter and Mr David Dick of the School's Workshop are also acknowledged for connecting the LEDs and fabricating the reactor vessels.

\section{References}

[1] Blankenship, R. E. (2002), Molecular Mechanisms of Photosynthesis, Blackwell Science Ltd, United Kingdom.

[2] Wang, C.-Y., Fu, C.-C. and Liu, Y.-C. (2007) 'Effects of using lightemitting diodes on the cultivation of Spirulina platensis ', Biochemical Engineering Journal, 37(1), pp. 21-25.

[3] Yan, C., Zhang, L., Luo, X. and Zheng, Z. (2013) 'Effects of various LED light wavelengths and intensities on the performance of purifying synthetic domestic sewage by microalgae at different influent $\mathrm{C} / \mathrm{N}$ ratios', Ecological Engineering, 51 pp. 24-32.

[4] Mehta, R., Deshpande, D., Kulkarni, K., Sharma, S. and Divan, D. (2008) 'LEDs - A competitive solution for general lighting applications', IEEE Energy 2030 Conference. Atlanta, Georgia, IEEE.

[5] Matthews, D. H., Matthews, H. S., Jaramillo, P. and Weber, C. L. (2009) IEEE International Symposium on Sustainable Systems and Technology. Tempe, AZ, USA, IEEE.

[6] Bracklow, U., Drews, A., Vocks, M. and Kraume, M. (2007) 'Comparison of nutrients degradation in small scale membrane bioreactors fed with synthetic/domestic wastewater', Journal of Hazardous Materials, 144(3), pp. 620-626.

[7] Borchardt, J. A. and Azad, H. S. (1968) 'Biological extraction of nutrients', Water Pollution Control Federation, 40(10), pp. 1739-1754.

[8] Lee, K. and Lee, C.-G. (2001) 'Effect of light/dark cycles on wastewater treatments by microalgae', Biotechnology and Bioresource Engineering, 6(3), pp. 194-199.

[9] APHA. (2005), Standard methods for the examination of water and wastewater, 21 st ed, APHA.

[10] Lee, R. E. (1999), Phycology, 3rd ed, Cambridge University Press, Cambridge.

[11] Ludwig, H. F., Oswald, W. J., Gotaas, H. B. and Lynch, V. (1951) 'Algae symbiosis in oxidation ponds I: growth characteristics of Euglena gracilis cultured in sewage', Sewage and industrial wastes, 23(11), pp. 1337-1355. 
[12] Van Den Hende, S., Vervaeren, H. and Boon, N. (2010) 'Industrial symbiosis: $\mathrm{C}, \mathrm{N}$ and $\mathrm{P}$ scavenging from sewage and flue gas with algal bacterial flocs', Journal of Biotechnology, 150(S), pp. 278.

[13] Philips, S., Laanbroek, H. J. and Verstraete, W. (2002) 'Origin, causes, and effects of increased nitrite concentrations in aquatic environments', Re/Views in Environmental Science \& Bio/Technology, 1(2), pp. 115-141. 